\title{
DysTS - An initiative to eradicate the inadequate treatment of Dyslexia in Sri Lanka
}

\author{
Zeinab Shabbir \\ E-mail address: zshabbir@gmail.com \\ eHealth Sri Lanka 2010,1(suppl.1):S28 \\ DOI: http://dx.doi.org/10.4038/sljbmi.v1i0.3586
}

Only the Abstract is available

\begin{abstract}
Dyslexia is a learning disability that causes difficulty in reading, writing and spelling. It prevents those affected from learning at the same level of ease and confidence as their non-dyslexic peers. There have been a few initiatives towards helping dyslexics in Sri Lanka, but a majority of them do not cater to individual needs. There is inadequate focus on the background and interests of each dyslexic, which should be key factors in designing their learning programmes. Additionally, the inability to objectively track the progress of dyslexics deters advancements in their educational and professional endeavours. Overall, it has been noted that due to their language difficulties, some dyslexics are still deprived of their basic social and educational rights. In order to obtain the requirements for a solution to address the above problem, interviews were conducted with officials and tutors of special schools which accentuated the need for a more resource-friendly teaching system.

Video-conferences with international medical professionals highlighted the impact of parental support in the progress of a dyslexic child. A total of 107 questionnaire responses stressed on the need for a computer-based teaching and tracking mechanism. Observation sessions and documents sampled emphasised on the need for an individualistic teaching approach. As such, the construction of a website as an automated teaching tool for dyslexics stood out as the ideal solution. A prototype of the solution was developed using Joomla, which is an open-source content management system integrated with My SQL, Apache and PHP. The solution incorporates concepts like multi-sensory teaching, verbal conceptualisation, phonetic awareness and confidence building into a website to positively aid the educational activities of dyslexic children. It also provides parental guidance, allows them to monitor the progress of their child and enables tutors to objectively evaluate the performance of each dyslexic. It was found that graphics and multimedia were effective in capturing and retaining the attention of dyslexic children. Parental involvement added to the learning enthusiasm of dyslexic children and increased the monitoring ability of tutors. Automated report-generation resulted in accurate performance tracking and allowed tutors to focus on other higher-priority activities.
\end{abstract}

Keywords - DysTS, Dyslexia, difficulty in reading, language difficulties, automated teaching tool 